\title{
Aterosclerosis en sujetos con periodontitis
}

NÉSTOR J. LÓPEZ1,a, ADRIANA CHAMORRO², MARCELO LLANCAQUEO³

\section{Association between atherosclerosis and periodontitis}

Background: Chronic inflammation and infections are involved in the development and progression of atherosclerotic vascular disease. Aim: To evaluate the association between periodontitis and early atherosclerosis. Material and Methods: Fifty-three subjects who received periodontal treatment and regular maintenance for at least 10 years, and 55 subjects with periodontitis but without a history of periodontal treatment were studied. Carotid artery intima-media wall thickness (CIMT) was measured with high-resolution B-mode ultrasonography. A blood sample was obtained to measure high sensitivity $C$-reactive protein, fibrinogen, lipoprotein cholesterol, leukocyte count and erythrocyte sedimentation rate. Covariates included age, gender, smoking, level of education, body mass index and physical activity. The benzoyl-DL-arginine-naphthylamide (BANA) test was used to determine the number of periodontal sites with periodontal pathogens. Results: CIMT value was significantly higher in subjects with periodontitis than those without it $(0.775 \pm 0.268$ and $0.683 \pm 0.131 \mathrm{~mm}$ respectively, $p=0.027$ ). C-reactive protein, leukocyte count and percentage of sites with periodontal pathogens were also significantly higher in subjects with periodontitis. Regression analysis identified age, periodontitis, and smoking as independent predictors of CIMT. Conclusions: These results suggest that untreated periodontitis is associated with early atherosclerotic carotid lesions and higher levels of inflammatory markers.

(Rev Med Chile 2011; 139: 717-724).

Key words: Atherosclerosis; Periodontitis; Risk factors.

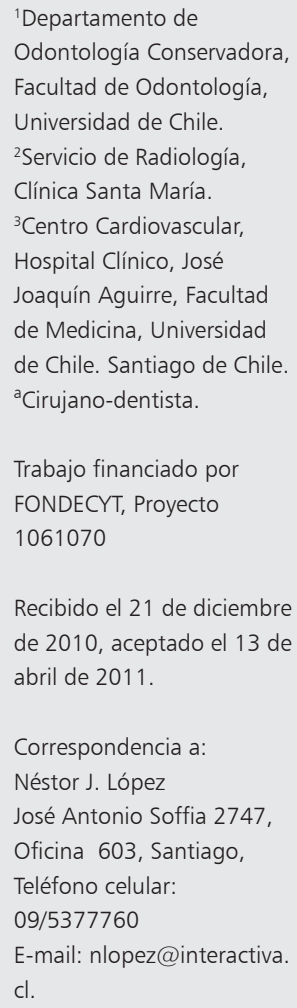

L as enfermedades cardiovasculares (ECV) son la primera causa de mortalidad en Chile, con $\checkmark$ una prevalencia de $28 \%{ }^{1}$. La ateromatosis es el sustrato anatomopatológico de las ECV y se ha demostrado que la inflamación e infecciones crónicas tienen un papel fundamental en la patogenia de la aterosclerosis ${ }^{2-4}$. La proteína C-reactiva (PCR), un marcador de la inflamación, añade un riesgo adicional a los factores de riesgo clásicos, como son hipertensión arterial, diabetes mellitus, dislipidemia y tabaquismo ${ }^{5}$. Sin embargo, los factores de riesgo clásicos de ECV explican sólo el $50 \%$ de las muertes por enfermedad coronaria ${ }^{6}$.

La periodontitis es una enfermedad inflamatoria crónica que destruye los tejidos periodontales causada por bacterias Gram negativas ${ }^{7}$. En los últimos años varios estudios han demostrado la asociación de periodontitis con mayor frecuencia de eventos cardiovasculares ${ }^{8-11}$ y de riesgo cardiovascular ${ }^{12}$. La periodontitis aumenta los niveles plasmáticos de mediadores de inflamación sistémica (MIS) asociados a aterosclerosis ${ }^{13,14}, \mathrm{y}$ origina bacteremias ${ }^{15}$. Se ha identificado patógenos periodontales en placas de ateroma ${ }^{16} \mathrm{y}$ endotelio de arterias coronarias ${ }^{17}$. Estudios experimentales sugieren que estos patógenos pueden estar involucrados en la patogénesis de la aterosclerosis ${ }^{18,19}$.

La medición del grosor de la íntima y media de las arterias carótidas (GIMC), usando ultrasonido, permite diagnosticar aterosclerosis subclínica ${ }^{20-22}$. 
Se ha demostrado asociación entre periodontitis $\mathrm{y}$ aterosclerosis temprana en sujetos sin signos de $\mathrm{ECV}^{23,24}$, y entre carga de patógenos periodontales y $\mathrm{GIMC}^{25}$, que disminuye significativamente después del tratamiento periodontal en sujetos $\sin \mathrm{ECV}^{26}$. Este último estudio es el único que ha demostrado el efecto del tratamiento de la periodontitis sobre la aterosclerosis temprana. $\mathrm{La}$ periodontitis es una enfermedad prevenible y de fácil tratamiento por lo que es importante determinar si su eliminación como factor contribuyente de aterosclerosis reduce el riesgo cardiovascular.

El objetivo de este estudio fue determinar la posible asociación entre tratamiento periodontal $\mathrm{y}$ aterosclerosis

\section{Material y Métodos}

Seleccionamos dos grupos de participantes. Los casos fueron sujetos con periodontitis que habían solicitado tratamiento entre marzo de 2007 y diciembre de 2008. La definición de caso incluyó: 1) Edad $\geq 50$ años sin historia de tratamiento periodontal; 2) Al menos 20 dientes presentes, de los cuales 6 debían tener 1 o más sitios con profundidad al sondaje $\geq 4 \mathrm{~mm}$ y pérdida de inserción $\geq 3 \mathrm{~mm}$ e inflamación gingival. Estas características son signos diagnósticos de periodontitis.

Los controles fueron sujetos que tuvieron periodontitis moderada a severa e historia de tratamiento periodontal. La definición de control incluyó: 1) Haber tenido periodontitis moderada a severa tratada al menos 10 años antes del estudio; 2) Haber recibido tratamiento de mantención periodontal al menos cada 6 meses, sin recurrencia de la periodontitis; 3 ) Tener radiografías dentales previas al tratamiento con pérdida ósea $\geq 30 \%$.

Los criterios de exclusión fueron: artritis reumatoidea u otras enfermedades crónicas cuyo tratamiento necesita uso regular de anti-inflamatorios o antibióticos.

Cincuenta y cinco sujetos identificados como controles fueron invitados a participar, y 53 aceptaron. Dos sujetos se excusaron por falta de tiempo para asistir a los exámenes. Cincuenta y cinco sujetos con periodontitis fueron invitados a participar y todos aceptaron. El Comité de Ética de la Facultad de Odontología aprobó el estudio y todos los participantes firmaron el consentimiento.

\section{Examen médico}

Las características socio-demográficas, factores de riesgo cardiovascular, y otros antecedentes médicos fueron investigados por el cardiólogo (M. LL.) que efectuó el examen médico de los participantes. Se efectuó una historia médica con registro de antecedentes familiares e individuales de riesgo cardiovascular. Se calculó el índice de masa corporal (IMC) (peso corporal/estatura en metros $^{2}$ ). Se midió la presión arterial sistólica y diastólica por duplicado con un aparato electrónico calibrado, con el paciente sentado. Se consideró hipertenso si usaba antihipertensivos, o tenía presión sistólica $\geq 140 \mathrm{~mm}$ y/o diastólica $\geq 90 \mathrm{~mm}$ de $\mathrm{Hg}$ en al menos dos ocasiones. Se determinó la historia de hipercolesteremia y el uso de medicamentos. La diabetes se identificó por el uso de hipoglicemiantes o glicemia $\geq 126 \mathrm{mg} / \mathrm{dL}$ en ayunas en dos mediciones. Los hábitos de tabaquismo se investigaron y se registró el uso pasado y presente de tabaco. El consumo de alcohol se categorizó por el equivalente al número de vasos de vino consumidos por semana. La actividad física y la situación laboral (trabajo remunerado actual o jubilado) también se registraron. La actividad física se definió como cualquier ejercicio físico en tiempo de ocio. Se determinaron los hábitos alimenticios por la frecuencia del consumo de vegetales por semana.

\section{Examen bucal}

El examen bucal incluyó la medición y registro de variables periodontales de cada diente con una sonda $\mathrm{N}^{\circ} 15$ de U. C.N, a saber, profundidad al sondaje, medición de pérdida de inserción y de sangrado gingival en 6 sitios de cada diente. Se efectuó un examen radiográfico de todos los dientes. El porcentaje de hueso alveolar perdido se midió con una regla de Schei usando la unión cemento-esmalte y la cresta ósea proximal como referencias.

\section{Detección de patógenos periodontales con Test BANA}

El test BANA (BANA Met LLC Washtenaw, Ann Arbor, Mi, US) detecta N-benzoyl-DL-arginina-2naphthylamida, una enzima que está presente sólo en Porphyromonas gingivalis, Treponema denticola, y Tannerella forsythensis ${ }^{27}$, patógenos importantes de la periodontitis. El test BANA se usó para identificar los sitios periodontales con estos patógenos. 
Se tomó una muestra de placa dental subgingival del sitio periodontal más profundo de cada cuadrante de la boca. La muestra se extendió sobre la parte inferior de la cinta de la tarjeta BANA y la parte superior de la cinta se humedeció con agua bidestilada esterilizada. La tarjeta BANA se dobló para hacer coincidir la mitad superior con la inferior, y se incubó a $55^{\circ} \mathrm{C}$ por 5 minutos. El color de la mitad superior de la cinta se registró como no coloreado (negativo) o azul (positivo).

\section{Procedimientos de laboratorio}

Se tomó una muestra de sangre después de un ayuno de 10 horas a cada participante. Antes de efectuarla se aplicó un cuestionario diseñado para identificar infecciones $u$ otras causas que pudieran afectar los MIS. Se determinó el número de leucocitos, velocidad de sedimentación globular (VSG) (método de Westergreen), niveles de glicemia, colesterol de lipoproteínas total (CT), colesterol de lipoproteínas de alta densidad (CHDL), de baja densidad (CLDL) y triglicéridos con métodos estándares de laboratorio (DiaSys Diagnostic Systems GmbH, Holzheim, Alemania). Se determinaron niveles séricos de fibrinógeno (método de Clauss) y de proteina C-reactiva (PCR) (Immunoturbidimetric assay, Cobas Integra, Roche Diagnostic, Mannheim, Alemania; límite de detección: 0,425 mg/dL).

\section{Medición del grosor de la intima y media de arterias carótidas}

Una radióloga experta y previamente calibrada (A. Ch.) que no conocía el grupo al que pertenecía el sujeto, usó ultrasonido en tiempo real (Siemens Sonoline Antares with VSX9-4, 9 and $13 \mathrm{MHz}$ transducers) para evaluar el GIMC. El examen se efectuó de acuerdo a la técnica descrita por Burke et $\mathrm{a}^{22}$. Brevemente, las carótidas de ambos lados se examinaron en el área proximal a $1 \mathrm{~cm}$ de la dilatación del bulbo carotídeo, la bifurcación de las carótidas ( $1 \mathrm{~cm}$ desde la división) y la carótida interna hasta $1 \mathrm{~cm}$ hacia distal de la división.

Se calculó el promedio de las mediciones de las arterias carótidas comunes, de la bifurcación y de las carótidas internas. El valor final del GIMC de cada sujeto fue el promedio de las seis mediciones ${ }^{22}$. Se registró también la presencia de placas de ateroma en las seis áreas examinadas. El criterio para definir placa ateromatosa fue el aumento de grosor localizado, mayor a $1 \mathrm{~mm}$ de la pared arterial, y con aumento de al menos 100\% del grosor comparado con los segmentos de las paredes adyacentes.

\section{Estadística}

Se calculó que una muestra de 50 sujetos en cada grupo tendría un poder de $80 \%$ para detectar una diferencia de GIMC de 0,11 con 0,20 mm de desviación estándar (DE), con un nivel de significación de 5\%. Se calculó el promedio de las mediciones de cada sujeto y luego se promediaron en cada grupo. La significación de las diferencias entre las variables continuas se analizaron con el test-t, y entre las variables categóricas con el test de $\chi^{2}$. Se efectuaron modelos de regresión lineal para multivariables usando GIMC como variable dependiente y periodontitis como variable categórica. Se confeccionaron modelos de regresión bivariables con los siguientes confundentes potenciales: edad, sexo, tabaquismo, nivel educacional, IMC, CT, CHD, y CLDL, presión diastólica y sistólica, diabetes, actividad física, consumo de vegetales, consumo de alcohol, uso de estatina y de aspirina. Las variables cuya asociación con GIMC mostraron un valor de $\mathrm{p}<0,25$ se incluyeron en modelos multivariables. Los datos de colesterol, P-CR, fibrinógeno, VSG y de leucocitos no tenían distribución normal y se transformaron logarítmicamente antes de los análisis. Los valores se describen en promedio $\pm \mathrm{DE}$ o porcentaje. $\mathrm{La}$ significación estadística se fijó en un valor de $\mathrm{p}<$ 0,050. Los análisis se efectuaron con el programa SYSTAT (versión 12.02, Illinois, USA).

\section{Resultados}

Las características demográficos y de riesgo cardiovascular de los participantes se muestran en la Tabla 1. La distribución del estado civil, de la situación laboral, del uso de estatinas y aspirina, de la actividad física y del consumo de vegetales fue similar en ambos grupos.

El grupo con periodontitis tenía significativamente menor promedio de edad, mayor proporción de hipertensos, fumadores, obesos y sujetos con menos actividad física. Las otras características tenían similar distribución en ambos grupos. Todos los hipertensos recibían terapia con medicamentos, y algunos sujetos con dislipidemia recibían estatinas. Los diabéticos tenían diabetes tipo 2 y recibían hipoglicemiantes. 
Tabla 1. Características de los sujetos con y sin periodontitis

\begin{tabular}{|c|c|c|c|}
\hline Característica & $\begin{array}{c}\text { Con periodontitis } \\
n=55\end{array}$ & $\begin{array}{c}\text { Sin periodontitis } \\
n=53\end{array}$ & $\mathbf{P}$ \\
\hline Edad & $58,32 \pm 4,23$ & $61,71 \pm 6,42$ & 0,036 \\
\hline Sexo/mujer & $29(52,72 \%)$ & $35(66,03 \%)$ & 0,22 \\
\hline Nivel educacional $>12$ años & $33 / 55 \quad 60 \%$ & $41 / 53 \quad 77,35 \%$ & 0,083 \\
\hline Historia familiar de ECV & $21(38,18 \%)$ & $25(47,16 \%)$ & 0,34 \\
\hline Historia familiar de diabetes & $27(49,09 \%)$ & $21(39,62 \%)$ & 0,32 \\
\hline Historia familiar de hipertensión & $33(60 \%)$ & $22(41,50 \%)$ & 0,055 \\
\hline Hipertensión & $30(54,54 \%)$ & $16(30,18 \%)$ & 0,010 \\
\hline Diabetes & $8(14,54 \%)$ & $5 \quad(9,43 \%)$ & 0,41 \\
\hline Presión arterial sistólica $\mathrm{mmHg}$ & $119,6 \pm 16,3$ & $117,6 \pm 13,2$ & 0,30 \\
\hline Presión arterial diastólica mmHg & $67,2 \pm 9,6$ & $65,2 \pm 7,9$ & 0,24 \\
\hline IMC $\left(\mathrm{kg} / \mathrm{m}^{2}\right)$ & $28,14 \pm 3,18$ & $25,15 \pm 3,94$ & 0,001 \\
\hline $\mathrm{IMC}>30\left(\mathrm{~kg} / \mathrm{m}^{2}\right)$ & $16(20,09 \%)$ & $6(11,32 \%)$ & 0,022 \\
\hline $\begin{array}{l}\text { Consumo de alcohol } \\
5-5 \text { vasos por semana } \\
>5 \text { vasos por semana }\end{array}$ & $\begin{array}{rr}31 & (56,36 \%) \\
3 & (4,54 \%)\end{array}$ & $\begin{array}{rc}39 & (73,58 \%) \\
0 & (0,0 \%)\end{array}$ & $\begin{array}{l}0,61 \\
0,085\end{array}$ \\
\hline No fumador & $18(32,72 \%)$ & $22(40 \%)$ & 0,55 \\
\hline Fumador actual & $19(34,54 \%)$ & $10(18,86 \%)$ & 0,037 \\
\hline Fumador previo & $18(32,72 \%)$ & $23(43,39 \%)$ & 0,34 \\
\hline
\end{tabular}

Tabla 2. Características periodontales

\begin{tabular}{|lccc|}
\hline Característica & Con periodontitis & Sin periodontitis & P \\
\hline Número de dientes perdidos & $4,89 \pm 3,96$ & $2,81 \pm 3,81$ & 0,007 \\
\hline Promedio de profundidad al sondaje (PS) (mm) & $3,74 \pm 0,76$ & $2,81 \pm 0,39$ & 0,0001 \\
\hline Porcentaje de sitios con sangramiento & $47,60 \pm 19,57$ & $12,84 \pm 9,82$ & 0,0001 \\
\hline Promedio de pérdida de inserción clínica (mm) & $3,91 \pm 1,18$ & $2,85 \pm 1,17$ & 0,0001 \\
\hline Porcentaje sitios con PS $\geq 4$ mm & $37,09 \pm 20,12$ & $4,78 \pm 5,61$ & 0,0001 \\
\hline Porcentaje superficies con placa & $74,83 \pm 23,44$ & $45,17 \pm 24,64$ & 0,0001 \\
\hline
\end{tabular}

Los casos tenían pobre estado periodontal y había diferencias altamente significativas entre casos y controles en todas las variables periodontales (Tabla 2). Los casos tenía mayor número de dientes cariados $(\mathrm{p}=0,046)$, menor número de controles dentales anuales $(\mathrm{p}=0,0001)$ y mayor porcentaje de sitios con pérdida de inserción $\geq 3 \mathrm{~mm}(\mathrm{p}=0,0001)$. Los controles tenían carac- terísticas periodontales compatibles con estado periodontal normal (Tabla 2). En los sujetos controles el tiempo promedio transcurrido entre el tratamiento y la fecha de ingreso al estudio fue $21,85 \pm 8,3$ años (rango 10 a 31). Los sujetos con periodontitis tenían mayores niveles de todos los MIS pero sólo P-CR y leucocitos fueron significativos. Los sujetos sin periodontitis tenían niveles 
Tabla 3. Marcadores bioquímicos

\begin{tabular}{|lccc|}
\hline Característica & Con periodontitis & Sin periodontitis & Valor de p \\
\hline Colesterol total de lipoproteínas (mg/dl) & $204,8 \pm 42,6$ & $205,7 \pm 33,6$ & 0,90 \\
\hline Colesterol LDL de lipoproteínas (mg/dl) & $122,3 \pm 37,6$ & $120,05 \pm 33,2$ & 0,74 \\
\hline Colesterol HDL de lipoproteínas (mg/dl) & $49,12 \pm 12,7$ & $66,53 \pm 17,96$ & 0,0001 \\
Triglicéridos (mg/dl) & $164 \pm 86$ & $108 \pm 51$ & 0,0001 \\
\hline Glicemia (mg/dl) & $104 \pm 44$ & $89,6 \pm 9,7$ & 0,020 \\
Grosor íntima y media (mm) & $0,775 \pm 0,268$ & $0,683 \pm 0,131$ & 0,027 \\
Número de placas de ateroma & $10(18,18)$ & $5(9,43)$ & 0,17 \\
P-CR (promedio mg/dL) & $3,28 \pm 2,89$ & $2,15 \pm 3,04$ & 0,052 \\
\hline P-CR logarítmica. mediana (rango intercuartil) & $0,342(0,01-0,628)$ & $0,01(0,01-0,301)$ & 0,030 \\
Fibrinógeno (promedio mg/dL) & $329 \pm 91$ & $305 \pm 64$ & 0,11 \\
Velocidad eritrosedimentación (mm a la hora) & $13,45 \pm 14,33$ & $10,90 \pm 7,71$ & 0,25 \\
\hline Número de leucocitos x mm ${ }^{3}$ & $7,16 \pm 1,61$ & $5,70 \pm 1,29$ & 0,0001 \\
\hline
\end{tabular}

Tabla 4. Análisis de regresión lineal múltiple de la relación entre GIMC como variable dependiente $\left(R^{2}=\mathbf{0 . 4 1 8}\right)$

\begin{tabular}{|lccccc|}
\hline Variable explicativa & Valor de $\beta \mathbf{2}$ & $\begin{array}{c}\mathbf{9 5 \%} \text { Intervalo } \\
\text { de confianza }\end{array}$ & $\begin{array}{c}\text { Error } \\
\text { estándar }\end{array}$ & Valor de t & Valor de $\mathbf{p}$ \\
\hline Constante & $-0,050$ & $-0,050-0,404$ & 0,179 & $-0,0280$ & 0,780 \\
\hline Edad & 0,012 & $0,016-0,009$ & 0,002 & 7,326 & 0,0001 \\
\hline Periodontitis & 0,068 & $0,133-0,002$ & 0,033 & 2,056 & 0,042 \\
\hline Tabaquismo & 0,064 & $-0,000-0,127$ & 0,032 & 1,995 & 0,049 \\
\hline IMC & 0,005 & $0,005-(-0,003)$ & 0,105 & 1,206 & 0,231 \\
\hline Colesterol total de lipoproteínas & $-0,001$ & $-0,002-0,001$ & 0,001 & $-1,146$ & 0,254 \\
\hline Colesterol LDL de lipoproteínas & 0,001 & $0,001-0,003$ & 0,001 & 1,800 & 0,075 \\
\hline Colesterol HDL de lipoproteínas & $-0,002$ & $-0,005-(-0,002)$ & 0,002 & $-0,935$ & 0,352 \\
\hline
\end{tabular}

significativamente mayores de CHDL (Tabla 3).

El test BANA fue positivo en 161 de 220 sitios $(73,18 \%)$ de los casos y en 89 sitios de 212 $(41,98 \%)$ de los controles $(\mathrm{p}=0,0001)$. No hubo asociación entre test BANA positivo y GIMC.

El promedio de GIMC fue significativamente mayor en los casos $(0,775 \pm 0,268 \mathrm{~mm}$, rango 0,48 a 1,03 ) que en los controles $(0,683 \pm 0,131$ $\mathrm{mm}$, rango 0,50 a $1,48 \mathrm{p}=0,027)$. Un mayor número de casos $(\mathrm{N}=10)$ que de controles $(\mathrm{N}=5)$ tenía placas de ateroma, pero la diferencia no fue significativa $(\mathrm{p}=0,17)$. Diecisiete sujetos con periodontitis $(30,90 \%)$ y nueve sujetos sin periodontitis $(16,98 \%)$ tenían grosor de carótidas mayor de $0,82 \mathrm{~mm}(\mathrm{p}=0,13)$.

El análisis de regresión lineal múltiple mostró que la edad, tabaquismo, y periodontitis eran predictores independientes asociados con GIMC (Tabla 4 ).

\section{Discusión}

En este estudio comparamos el GIMC, los niveles de MIS y la prevalencia de sitios con patógenos periodontales en sujetos que habían recibido tra- 
tamiento periodontal y mantención adecuada por lo menos 10 años, con sujetos con periodontitis no tratada, y se identificó a la periodontitis como un predictor independiente del GIMC. Las características de los casos indican que tenían periodontitis severa con más de $60 \%$ de los sitios con $\geq 3 \mathrm{~mm}$ pérdida de inserción. Se ha definido la periodontitis severa por la presencia de $\geq 60 \%$ sitios con $\geq 3$ $\mathrm{mm}$ de pérdida de inserción clínica, y se ha encontrado que este nivel de periodontitis está asociado con varias enfermedades sistémicas, incluyendo enfermedad arterial coronaria ${ }^{28,29}$. Como criterio diagnóstico de periodontitis usamos el número de sitios con profundidad al sondaje $\geq 4 \mathrm{~mm}$ y no con sólo pérdida de inserción, porque el número de sitios con lesiones periodontales representa mejor la carga de inflamación e infección periodontal. La pérdida de inserción clínica debida a periodontitis es generalmente irreversible y refleja la pérdida de inserción histórica más que el estado de inflamación. Por lo demás, en sujetos mayores de 45 años, como los del presente estudio, es común cierto grado de pérdida de inserción periodontal debido a otras causas, como el cepillado traumático de dientes y el efecto de la edad. Los sujetos que habían recibido tratamiento habían tenido periodontitis moderada a severa, por lo que tenían un número de sitios con pérdida de inserción $\geq 3$ $\mathrm{mm}$ cuando ingresaron al estudio, que es mayor a lo esperado en sujetos sin periodontitis.

Algunos autores ${ }^{30}$ han sugerido que la asociación entre periodontitis y ECV puede deberse a confundentes como insuficiente control del nivel socioeconómico y de estilos de vida. Para controlar efectos confundentes incluimos estado civil, nivel educacional, situación laboral, actividad física, hábitos de dieta y consumo de alcohol. Algunos de estos factores eran significativamente más frecuentes en el grupo con periodontitis pero ninguno de ellos estaba asociado con el GIMC. Usamos el nivel de educación como indicador de nivel socioeconómico por las limitaciones de usar el ingreso económico como indicador. Además todos los sujetos del estudio eran pacientes de práctica privada, lo que hace más probable un similar nivel socioeconómico de los participantes.

El nivel alto de lipoproteinas, hipertensión, tabaquismo, obesidad y diabetes son factores de riesgo de enfermedad coronaria y aterosclerosis ${ }^{31}$. En el grupo con periodontitis había una significativa mayor proporción de obesos, fumadores e hipertensos, pero sólo el tabaquismo estaba asociado con el GIMC. Todos los hipertensos estaban recibiendo tratamiento, lo que explica los niveles similares de presión sistólica y diastólica en sujetos con y sin periodontitis. Se ha demostrado fuerte relación entre colesterol LDL, HDL y triglicéridos y GIMC $^{32}$ que no se encontró en el presente estudio probablemente porque los niveles de CT y LDL, eran similares en ambos grupos. Los pacientes con periodontitis tenían niveles significativamente mayores de triglicéridos que los sin periodontitis, y más bajo nivel de CHDL, que puede ser el resultado de alteraciones metabólicas descritas en la periodontitis ${ }^{33,34}$.

Un GIMC $\geq 0,82 \mathrm{~mm}$ se ha asociado con enfermedad coronaria e infarto cerebral ${ }^{35,36}$. En nuestro estudio había más sujetos con periodontitis con GIMC $\geq 0,82 \mathrm{~mm}$ y con placas de ateroma que en sujetos sin periodontitis. Aún cuando estas diferencias no fueron significativas, muestran una tendencia de mayor prevalencia de aterosclerosis asociada a periodontitis.

Concentraciones de P-CR $>3 \mathrm{mg} / \mathrm{L}$ son un buen predictor de mortalidad por causa cardiovascular $^{37} \mathrm{y}$ de riesgo de accidente cerebrovascular ${ }^{38}$, y estudios recientes indican que P-CR tiene un rol en las lesiones ateromatosas ${ }^{39}$. Nuestros pacientes con periodontitis tenían niveles de P-CR y de leucocitos significativamente mayores que los sujetos sin periodontitis como se ha demostrado ${ }^{40}$. Se ha sugerido que niveles altos de MIS pueden explicar la conexión entre periodontitis y riesgo cardiovascular ${ }^{41}$.

El test BANA es una prueba sensitiva para determinar la presencia de al menos uno de los tres patógenos periodontales que identifica. Tiene $92 \%$ de sensibilidad y $70 \%$ de especificidad comparado con sondas de $\mathrm{ADN}$ y con reactivos inmunológicos policlonales para la identificación de bacterias periodontales ${ }^{27}$. Los sujetos con periodontitis tenían una proporción significativamente mayor de sitios BANA positivos, indicando que la carga de infección periodontal era mayor en ellos que en sujetos sin periodontitis.

El modelo de regresión lineal mostró que la edad fue el predictor más fuerte de GIMC, aún cuando la edad de los sujetos con periodontitis era significativamente menor que la de los sin periodontitis.

Las limitaciones del presente estudio son las propias del diseño de casos y controles relacionado 
con la imposibilidad de terminar la relación temporal entre periodontitis y aterosclerosis, ya que es un estudio transversal. Un estudio longitudinal para determinar esta asociación es complejo dado que se trata de dos enfermedades de larga evolución, y razones éticas impiden dejar sin tratar por largo tiempo a pacientes con periodontitis. Aún cuando en el estudio se determinaron todos los factores de riesgo de ECV conocidos, con excepción del estrés, existe la posibilidad que el tamaño muestral utilizado pudiera no tener el poder estadístico suficiente para identificar efectos confundentes.

Los resultados del presente estudio concuerdan con otros ${ }^{23-26}$ que han demostrado asociación entre periodontitis y aterosclerosis subclínica, enfatizando la importancia del diagnóstico precoz y tratamiento de periodontitis, especialmente en personas con riesgo cardiovascular.

\section{Referencias}

1. Instituto Nacional de Estadísticas. Estadísticas Vitales. Informe anual 2007, Publicado 16 dic. 2009, p. 31,32. Consultado el 30 de octubre de 2010 en www.ine.cl.

2. Danesh J, Collins R, Peto R. Chronic infections and coronary heart disease: is there a link? Lancet 1997; 350: 430-6.

3. Nieto FJ, Adam E, Sortie P, Farzadegan H, Melnick JL, Comstock GW, et al. Cohort study of cytomegalovirus infection as a risk factor for carotid intimal-media thickening, a measure of subclinical atherosclerosis. Circulation 1996; 94: 922-92.

4. Libby P, Ridker PM, Maseri A. Inflammation and atherosclerosis. Circulation 2002; 105: 1135-43.

5. Libby P. The Vascular Biology of Atherosclerosi. En: Braunwald's Heart Disease: A Textbook of Cardiovascular Medicine, Capítulo 38, 8ª ed. 2007, p. 987-1001.

6. Futterman LG, Lember L. Fifty per cent of patients with coronary artery disease do not have any of the conventional risk factors. Am J Crit Care 1998; 7: 240-4.

7. Concensus Report for Periodontal Diseases: pathogenesis and microbial factors. Ann Periodontol 1996; 1 : 926-32.

8. DeStefano F, Anda RF, Kahn HS, Williamson DF, Russell CM. Dental disease and risk of coronary heart disease and mortality. BMJ 1993; 306: 688-91.

9. Mattila KJ, Valtonen VV, Nieminen M, Huttunen JK. Dental infection and the risk of new coronary events: prospective study of patients with documented coronary artery disease. Clin Infect Dis 1995; 20: 588-92.

10. Morrison HI, Ellison LF, Taylor GW. Periodontal disease and risk of fatal coronary heart and cerebrovascular disease. J Cardiovasc Risk 1999; 6: 7-11.

11. Beck J, García R, Heiss G, Vokonas PS, Offenbacher S. Periodontal disease and cardiovascular disease. J Periodontol 1996; 67 (10 Suppl): 1123-37.

12. Janket S, Baird AE, Chuang S-K, Jones J. Meta-analysis of periodontal disease and risk of coronary heart disease and stroke. Oral Surg Oral Med Oral Pathol Oral Radiol Endod 2003; 95: 559-69.

13. Glurich I, Grossi S, Albini B, Ho A, Shah R, Zeid M, et al. Systemic inflammation in cardiovascular and periodontal disease: comparative study. Clin Diagn Lab Immunol 2002; 9: 425-32.

14. Slade GD, Ghezzi EM, Heiss G, Beck JD, Riche E, Offenbacher S. Relationship between periodontal disease and C-reactive protein among adults in the Atehrosclerosis Risk in Communities Study. Arch Int Med 2003; 163: 1172-81.

15. Forner L, Larsen T, Killian M, Holmstrup P. Incidence of bacteremia after chewing, tooth brushing and scaling in periodontal inflammation. J Clin Periodontol 2006; 33: 401-7.

16. Haraszthy VI, Zambon JJ, Trevisan M, Zeid M, Genco RJ. Identification of periodontal pathogens in atheromatous plaques. J Periodontol 2000; 71: 1554-60.

17. Desphande RG, Khan MB, Genco CA. Invasion of aortic and heart endothelial cells by Porphyromonas gingivalis. Infec Immun 1998; 66: 5337-43.

18. Giacona MB, Papapanou PN, Lamster IB, Rong LL, D'Agati VD, Schmidt AM, et al. Porphyromonas gingivalis induces its uptake by human monocytes/macrophages and promotes foam cell formation in vitro. FEMS Microbiol Lett 2004; 241: 95-101.

19. Roth GA, Moser B, Roth-Walter F, Giacona MB, Harja E, Papapanou PN, et al. Infection with a periodontal pathogen increases mononuclear cell adhesion to human aortic endothelial cells. Atherosclerosis 2007; 190: 271-81.

20. Grobbee DE, Bots ML. Carotid artery intima-media thickness as an indicator of generalized atherosclerosis. J Intern Med 1994; 236: 567-73.

21. Salonen JT, Salonen R. Ultrasound B-mode imaging in observational studies of atherosclerotic progression. Circulation 1993; 87: 1156-65.

22. Burke GL, Evans GW, Riley WA, Sharret AR, Howard G, Barnes RW, et al. Arterial wall thickness is associated with prevalent cardiovascular disease in middle-aged adults. Stroke 1995; 26: 386-91.

23. Beck JD, Elter JR, Heiss G, Couper D, Mauriello SM, 
Offenbacher S. Relationship of periodontal disease to carotid artery intima-media wall thickness. The atherosclerosis risk in communities (ARIC) Study. Arterioscler Thromb Vasc Biol 2001; 67: 1816-22.

24. Soder PO, Soder B, Nowak J, Jogestrand T. Early carotid atherosclerosis in subjects with periodontal diseases. Stroke 2005; 36: 1195-200.

25. Desvarieux M, Demmer RT, Rundek T, Boden-Albala B, Jacobs DR, Sacco RL, et al. Periodontal microbiota and carotid intima-media thickness. The oral infections and vascular disease epidemiology Study (INVEST). Circulation 2005; 111: 576-82.

26. Piconi S, Trabattoni D, Luraghi C, Perilli E, Borelli M, Pacei M, et al. Treatment of periodontal disease results in improvements in endothelial dysfunction and reduction of the carotid intima-media thickness. FASEB J 2009; 23: 1-9.

27. Loesche WJ, Lopatin DE, Stoll J, van Poperin N, Hujoel PP. Comparison of various detection methods for periodontopathic bacteria: can culture be considered the primary reference standard? J Clin Microbiol 1992; 30: 418-26.

28. Offenbacher S, Katz V, Fertik G, Collins J, Boyd D, Maynor G, et al. Periodontal infection as a possible risk factor for preterm low birth weight. J Periodontol 1996; 67: 1103-13.

29. Beck J, García R, Heiss G, Vokonas PS, Offenbacher S. Periodontal disease and cardiovascular disease. J Periodontol 1996; 67: 1123-37.

30. Hujoel PP, Drangsholt M, Spiekerman C, DeRouen TA. Periodontitis-systemic disease associations in the presence of smoking-causal or coincidental? Periodontology 2000 2002; 30: 51-60.

31. Grundy SM, Pasternak R, Greenland P, Smtih S Jr, Fuster V. Assessment of cardiovascular risk by use of multiplerisk-factor assessment equations: a statement for healthcare professionals from American Heart Association and the American College of Cardiology. Circulation 1999; 100: 1481-92.

32. Inter-Society Commission for Heart Disease Resources,
Atherosclerosis Study Group and Epidemiology Study Group: Primary prevention of the atherosclerotic diseases. Circulation 1970; 42 (Suppl): A55-A95.

33. Katz J, Sharabi Y, Chauasho G. On the association between hypercholesterolemia, cardiovascular disease and severe periodontal disease. J Clin Periodontol 2001; 28: 865-8.

34. Pussinen PJ, Jauhiainen M, Vilkuna-Rautiainen T, Sundvall J, Vesanen M, Mattila K, et al. Periodontitis decreases the antiatherogenic potency of high density lipoprotein. J Lipid Res 2004; 45: 139-47.

35. O’Leary D, Polak J, Kronmal RA, Manolio T, Burke G, Wolfson SJ. Carotid-artery intima and media thickness as a risk factor of myocardial infarction and stroke in older adults: Cardiovascular Health Study Collaborative Research Group. N Engl J Med 1999; 340: 14-22.

36. Chambless L, Folsom A, Clegg L, Sharrey A, Shahar E, Nieto F, et al. Carotid wall thickness is predictive of incident clinical stroke: the atherosclerotic risk in communities Study. Am J Epidemiol 2000; 15: 478-48.

37. Mendall MA, Strachan DP, Butland BK, Mendall MA, Strachan DP. C-reactive protein relation to total mortality, cardiovascular mortality and cardiovascular risk factors in men. Eur Heart J 2000; 21: 1584-159.

38. Curb JD, Abbott RD, Rodríguez BL, Sakkinen P, Popper JS, Yano K, et al. C-reactive protein and the future risk of thromboembolic stroke in healthy men. Circulation 2003; 107: 2016-20.

39. Blake GJ, Ridker PM. Inflammatory biomarkers and cardiovascular risk prediction. J Intern Med 2002; 252: 283-94.

40. Slade GD, Offenbacher S, Beck JD, Heiss G, Pankow JS. Acute-phase inflammatory response to periodontal disease in the US population. J Dent Res 2000; 79: 49-57.

41. Wu T, Trevisan M, Genco RJ, Falkner KL, Dorn JP, Sempos CT. Examination of the relation between periodontal health status and cardiovascular risk factors: serum total and high density lipoprotein cholesterol, C-reactive protein, and plasma fibrinogen. Am J Epidemiol 2000; 151:273-82. 